ARTICLE

https://doi.org/10.1038/s41467-019-13043-2

\title{
An open source automated tumor infiltrating lymphocyte algorithm for prognosis in melanoma
}

\author{
Balazs Acs (1) 1,2, Fahad Shabbir Ahmed (1) 1, Swati Gupta1, Pok Fai Wong1', Robyn D. Gartrell3, \\ Jaya Sarin Pradhan ${ }^{4}$, Emanuelle M. Rizk ${ }^{5}$, Bonnie Gould Rothberg ${ }^{6}$, Yvonne M. Saenger ${ }^{5}$ \& David L. Rimm (1) 1,6*
}

Assessment of tumor infiltrating lymphocytes (TILs) as a prognostic variable in melanoma has not seen broad adoption due to lack of standardization. Automation could represent a solution. Here, using open source software, we build an algorithm for image-based automated assessment of TILs on hematoxylin-eosin stained sections in melanoma. Using a retrospective collection of 641 melanoma patients comprising four independent cohorts; one training set $(N=227)$ and three validation cohorts $(N=137, N=201, N=76)$ from 2 institutions, we show that the automated TIL scoring algorithm separates patients into favorable and poor prognosis cohorts, where higher TILs scores were associated with favorable prognosis. In multivariable analyses, automated TIL scores show an independent association with disease-specific overall survival. Therefore, the open source, automated TIL scoring is an independent prognostic marker in melanoma. With further study, we believe that this algorithm could be useful to define a subset of patients that could potentially be spared immunotherapy.

\footnotetext{
${ }^{1}$ Department of Pathology, Yale School of Medicine, New Haven, CT 06510, USA. ${ }^{2}$ Department of Oncology and Pathology, Karolinska Institutet, Stockholm, Sweden. ${ }^{3}$ Department of Medicine, Division of Hematology/Oncology, Columbia University Medical Center/New York Presbyterian, New York, NY, USA. ${ }^{4}$ Department of Pathology and Cell Biology, Division of Oral and Maxillofacial Pathology, Columbia University Irving Medical Center/New York Presbyterian, New York, NY, USA. ${ }^{5}$ Department of Medicine, Division of Hematology/Oncology, Columbia University Irving Medical Center/New York Presbyterian, New York, NY, USA. ${ }^{6}$ Department of Medicine, Yale School of Medicine, New Haven, CT 06510, USA. *email: david.rimm@yale.edu
} 
T he development and progression of malignant tumors requires interaction with other cells in the tumor microenvironment, including immune cells ${ }^{1}$. Due to altered protein expression by tumor cells, the immune system can recognize malignancy and induce an immune response ${ }^{2,3}$. Growing evidence from several studies supports that assessment of tumor-infiltrating lymphocytes (TIL) has prognostic significance in many tumor types ${ }^{4}$. Recent studies have shown that immunotherapy in the adjuvant setting benefits only one in five patients ${ }^{5,6}$. These studies show significant toxicities including one in five with significant hypothyroidism and one in hundred fatalities ${ }^{5,6}$ These observations warrant the introduction of a prognostic test that can determine which patients can be spared treatment.

TILs have traditionally been scored semi-quantitatively on standard hematoxylin and eosin (H\&E)-stained section as absent, non-brisk, or brisk ${ }^{7}$. Some departments use a fourtier grading system that includes both TIL distribution and density $^{8}$. These scoring methods have been established for many years, but have not seen broad adoption in clinical decisionmaking due to lack of standardization between institutions and concerns regarding reproducibility between pathologists 9 . In breast cancer, the International TIL working group has put serious efforts on the standardization of TILs scoring and published a guideline that is made for visual evaluation of HE sections by pathologists ${ }^{10,11}$. Although the robustness of the TIL guideline has been shown in international ring trials ${ }^{12}$, it is unlikely that a subjective method will be sufficiently accurate and reproducible to be used to select patients to be spared from therapy ${ }^{13}$. Digital-image analysis (DIA) may present a solution to this problem. DIA can facilitate the analysis of complex spatial patterns, and could provide standardized metrics for rigorous validation ${ }^{13}$. Classical segmentation and neural networks approaches have been applied widely in various DIA platforms to overcome cell classification challenges ${ }^{14,15}$. DIA platforms are able to evaluate TILs, but no study has published yet to show robustness of automated TIL scoring in melanoma. Therefore, we built an algorithm (called eTIL\%) for image-based, automated assessment of tumor-infiltrating lymphocytes (TILs) on H\&Estained sections in melanoma.

\section{Results}

Performance of automated TIL scoring in TMA cohorts. The best threshold with statistical significance after cross-validation in Xtile occurred at $16.6 \%(p=0.01)$. The automated TIL scoring algorithm showed high eTIL\% patients showed statistically significantly better disease-specific overall survival (DSOS) compared with low eTIL\% (Log rank $p=0.007 ; \mathrm{HR}=0.420, \mathrm{CI}=$ $0.220-0.802$; Fig. 1a). Higher TILs scores were associated with favorable prognosis. In contrast, traditional pathologist-read visual assessment of TILs, modified to be assessed on a TMA, failed to distinguish patient cohorts with different DSOS (Log rank $p=0.821 ; \mathrm{HR}=0.871, \mathrm{CI}=0.461-1.646$; Fig. $1 \mathrm{~b}$ ). This same cohort was also tested for expression of CD4, CD8, and $\mathrm{CD} 20$ using quantitative fluorescent methods ${ }^{16}$. These results show a weak correlation of each of these lymphocytic subtypes to eTIL\% (Fig. 1c-e), but none were prognostic, although CD4 trended toward, but did not reach significance. We found a significant relationship and fair correlation between eTILs and the sum of CD4 and CD8 (Spearman $r=0.466, p<0.001$ ). Using the $16.6 \%$ cut point defined in cohort \#1 on TMA images from cohorts \#2 and \#3, eTIL\% separated patients into favorable and unfavorable prognostic subsets (cohort \#2: Log rank $p<$ $0.001 ; \mathrm{HR}=0.397, \mathrm{CI}=0.242-0.651$; Fig. 2a; cohort \#3: Log rank $p=0.002 ; \quad \mathrm{HR}=0.409, \quad \mathrm{CI}=0.226-0.741 ; \quad$ Fig. $\quad 2 b) . \quad$ The clinicopathological factors were prognostic in univariate analysis (Supplementary Table 1). We also investigated the association between eTIL\% and the clinicopathological factors. eTIL\% score was higher in cases with absent ulceration and smaller tumor depth in cohorts \#1, \#2, and \#3. No significant association was found between eTIL\% and any clinicopathological factor in cohort \#4 (Supplementary Fig. 1).

Performance of automated TIL scoring in whole-slide cohorts. In the clinical setting, assessments must be done on whole-tissue sections. So, we applied the NN192 algorithm to every field of view on whole-slide images from cohorts \#2 and \#4. Again eTIL\% high, above the $16.6 \%$, showed significantly favorable DSOS in both cohorts (cohort \#2: Log rank $p<0.001$; $\mathrm{HR}=0.119$, $\mathrm{CI}=$ $0.057-0.245$, Fig. $2 c)$ and cohort $\# 4(\log$ rank $p=0.036$; HR $=$ $0.391, \mathrm{CI}=0.157-0.974$; Fig. $2 \mathrm{~d}$ ). The eTIL\% score remained significant in both cohorts even after adjusting for age, sex, ulceration, stage (using the system concurrent with tissue collection dates), Breslow depth, Clarke levels, and location of primary tumor (Table 1). We found significant difference and low reproducibility between the corresponding TMA and WSI cases regarding eTIL\% scores (Supplementary Fig. 2).

\section{Discussion}

Although it has long been acknowledged that TILs might provide prognostic and predictive information in melanoma ${ }^{11,17-19}$, it has not been widely adopted for clinical melanoma management due to inter-operator and inter-institutional variability ${ }^{20}$. Key contributors to variability include both preanalytical and analytical steps, and most significantly, lack of reproducibility in scoring ${ }^{13,21}$. To address the issue of subjective variance, studies have been published proposing TIL quantification using convolutional networks on imagebased immunohistochemistry (IHC)-stained sections in gastric, breast, prostate, and colon cancer ${ }^{22-24}$. However, in these investigations, the TIL quantification relies on the detection of IHC markers, such as CD3 and CD8 and in some cases, depends on their localization with respect to the leading edge of the tumor ${ }^{25}$. In our previous work, we showed that digital-image analysis of CD8 detection is associated with anti-PD-1 response in metastatic melanoma, but is not prognostic ${ }^{16}$. Here, the prognostic value of the eTIL\% may arise from the combination of a range of lymphocytes combined with the normalization by the number of adjacent melanocytes. While subtyping of TILs may become important in immunotherapy prediction, also requiring image analysis, a combination of eTIL\% and quantitative CD8 might be the best approach for finding patients that can be spared immunotherapy in the adjuvant setting. In our study, we found a significant relationship and weak-fair correlation between eTILs and CD4, CD8 and CD20 expression. A possible explanation could be immune heterogeneity in the tumor as the $\mathrm{HE}$ and $\mathrm{CD} 4, \mathrm{CD} 8, \mathrm{CD} 20 \mathrm{IF}$ stainings were not performed on serial sections. Another possible explanation is that CD4, CD8, and CD20 (immune markers) scores were calculated differently during the automated quantitative fluorescent investigation. Regarding immune markers, scores were calculated in terms of the number of cells positive for the marker of interest as a percentage of the cell population in which it was measured, while eTIL\% was defined as (TILs/TILs + tumor cells) $\times 100$.

Detection of TILs might provide a cost-effective and robust prognostic marker, especially when no additional molecular tests are available. In a recent study by Heindl et al., the prognostic potential of automated TIL scoring was investigated in ER-positive $(+)$ breast cancer ${ }^{26}$. The authors used cell segmentation-classification approach for DIA on H\&E-stained sections, and they found that immune spatial clustering scores 
a

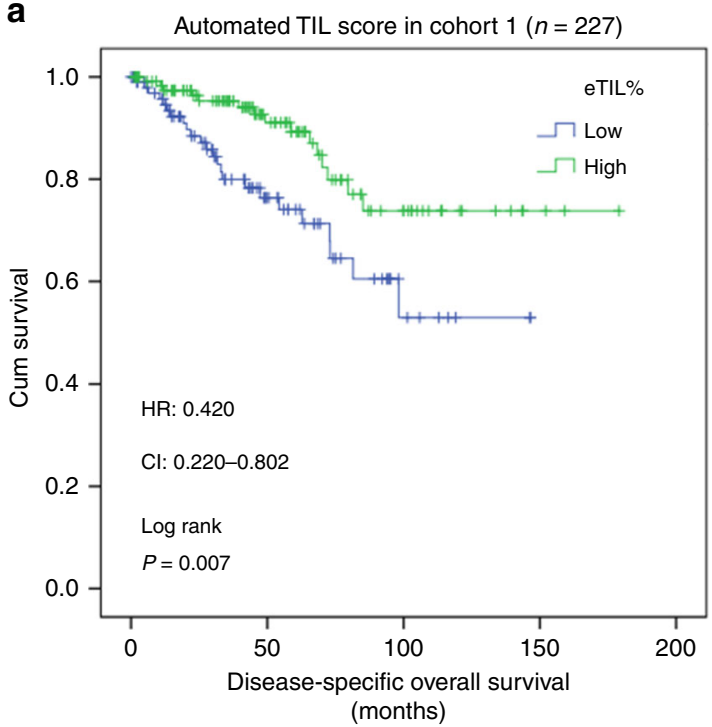

C

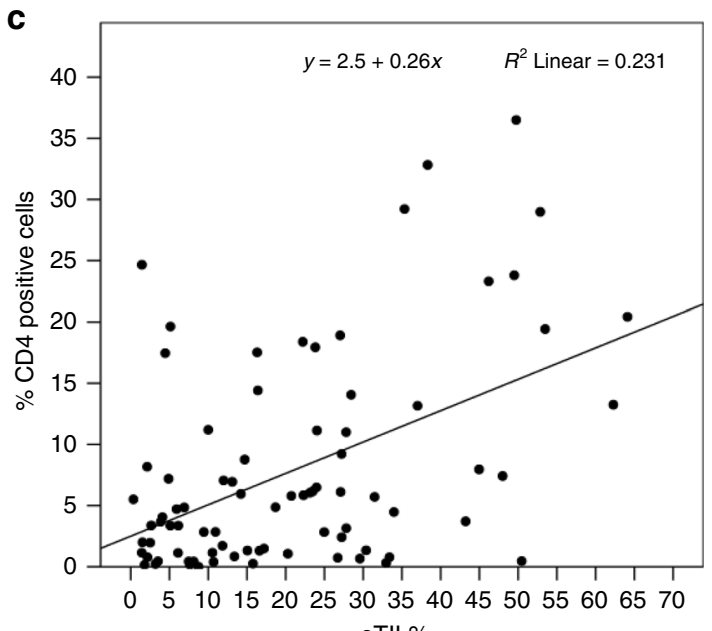

eTIL\%

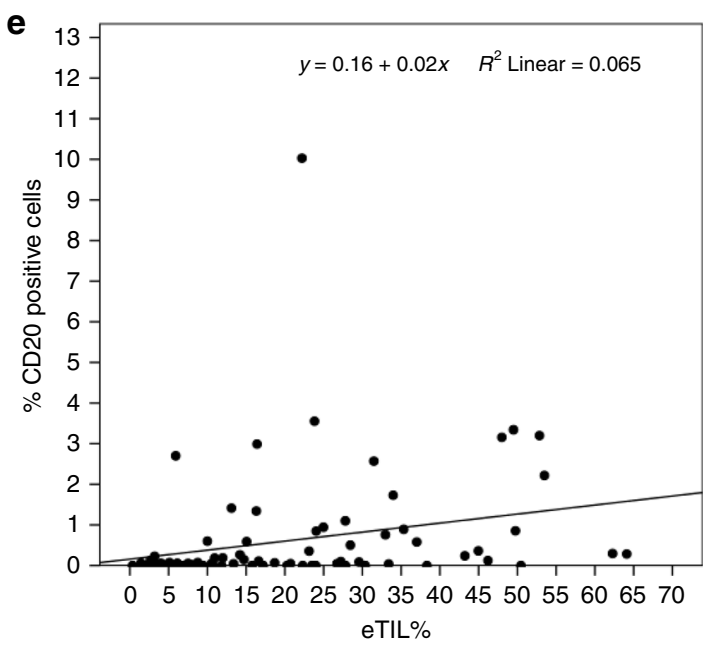

b

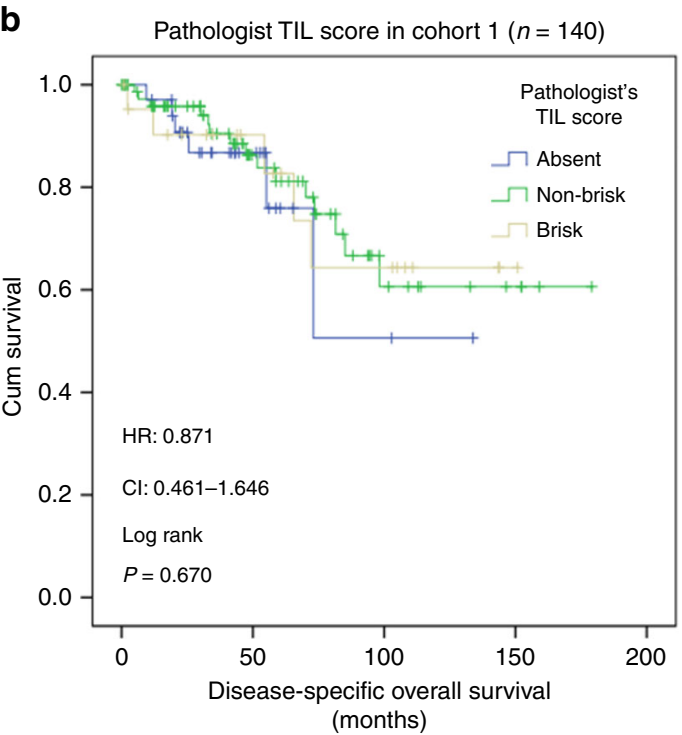

d

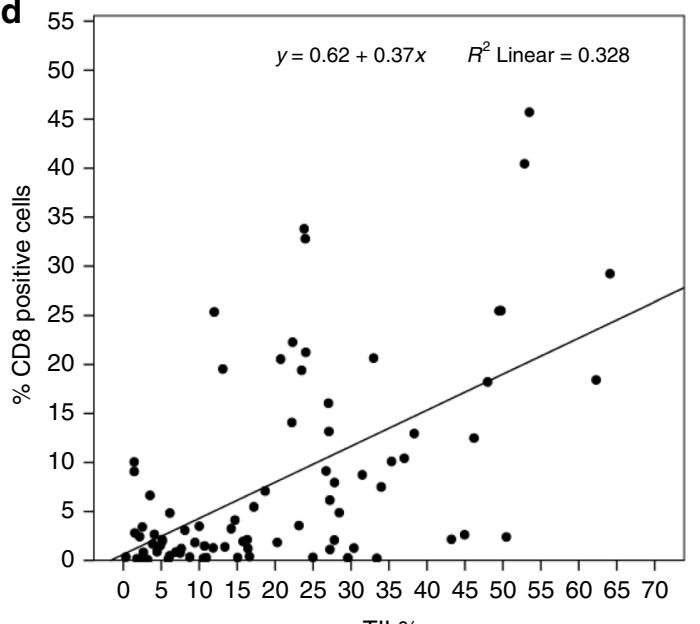

eTIL\%

f

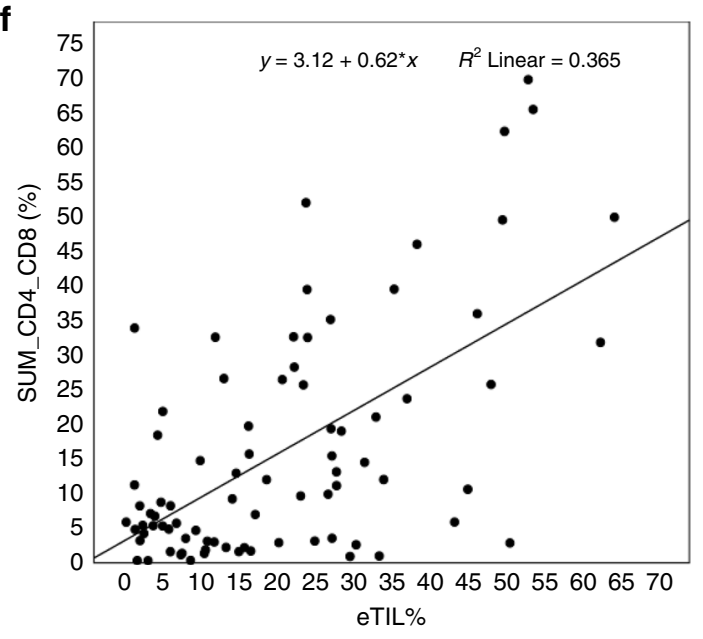

Fig. 1 Tumor-infiltrating lymphocytes (TIL) scores in cohort \#1. The prognostic potential of automated TIL scores (a) and pathologist's TIL scores (b) in cohort \#1. Correlation between eTIL\% scores and CD4 positive (+), CD8+, and CD20+ immune cells measured by quantitative immunofluorescence (c-f).

obtained by DIA were linked with poor recurrence-free survival after endocrine therapy in ER+ breast cancer ${ }^{26}$. In our study, we used a similar approach, unsupervised nuclei segmentation followed by neural network machine-learning-based cell classification. The advantage of segmentation-based object detection is that it requires relatively smaller of training sets $^{13,27,28}$. On the other hand, segmentation sensitivity and classification accuracy are subject to biological and technical 
a

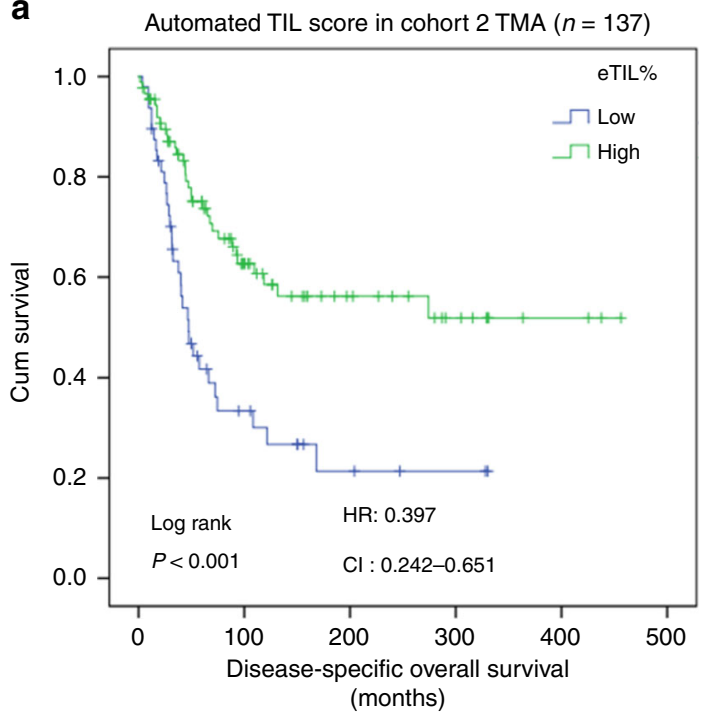

C

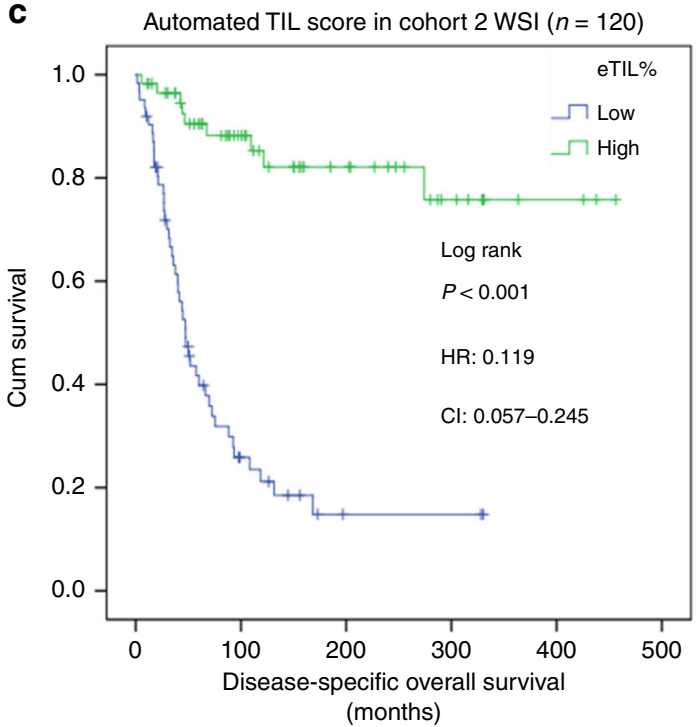

b

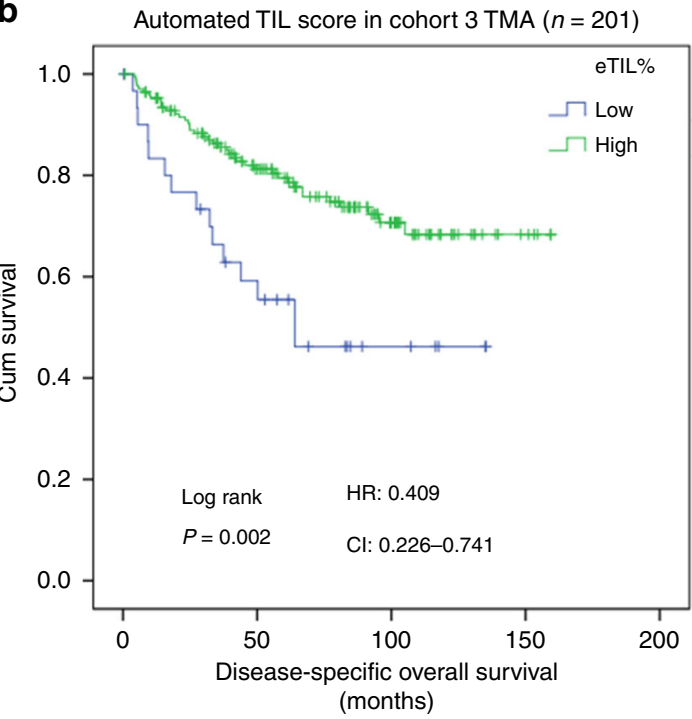

d

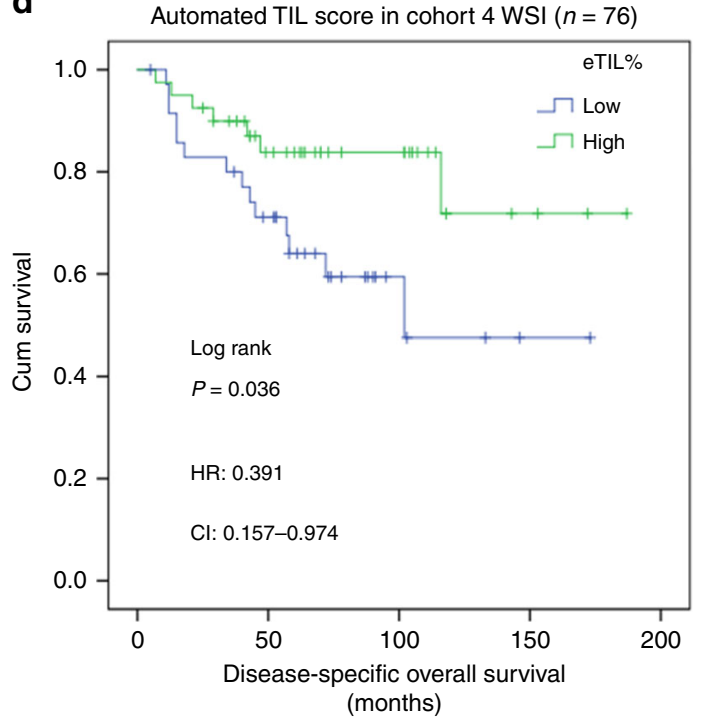

Fig. 2 Validation of automated tumor-infiltrating lymphocytes (TIL) algorithm in three independent cohorts. The prognostic potential of automated TIL scores performed on tissue microarray (TMA slides) $(\mathbf{a}, \mathbf{b})$ and whole slides $(\mathbf{c}, \mathbf{d})$ in cohorts \#2, \#3, and \#4.

Table 1 Multivariate Cox-regression analysis of eTIL\% score and the clinicopathological factors in whole-slide cohorts \#2 and \#4 regarding disease-specific overall survival.

Prognostic factor

Age

Age

Sex

Tumor depth

Clarke levels

Ulceration

Stage

Location of primary tumor

Automated TIL score

\begin{tabular}{lll}
$\begin{array}{l}\text { Cohort \#2 } \\
(\boldsymbol{n}=\mathbf{1 2 0})\end{array}$ & & \\
\hline HR & $\mathbf{9 5 \%} \mathbf{~ C l}$ & $\boldsymbol{p}$-value \\
\hline 0.985 & $0.958-1.013$ & 0.289 \\
1.601 & $0.876-2.927$ & 0.126 \\
1.156 & $0.747-1.788$ & 0.516 \\
1.369 & $0.708-2.645$ & 0.350 \\
1.190 & $0.623-2.272$ & 0.599 \\
1.389 & $0.990-1.948$ & 0.057 \\
NA & NA & NA \\
0.143 & $0.063-0.326$ & $<0.001$
\end{tabular}

both TMA and whole-slide sections. We found significant prognostic potential of automated TIL scores in all the validation sets.

There are a number of limitations to this work. Most significantly, the cohorts were all retrospectively collected, including

\begin{tabular}{lll}
$\begin{array}{l}\text { Cohort \#4 } \\
(\boldsymbol{n}=\mathbf{7 6})\end{array}$ & & \\
\hline HR & $\mathbf{9 5 \%} \mathbf{~ C l}$ & $\boldsymbol{p}$-value \\
\hline 1.014 & $0.979-1.049$ & 0.446 \\
0.497 & $0.129-1.914$ & 0.310 \\
1.441 & $0.514-4.043$ & 0.488 \\
NA & NA & NA \\
1.397 & $0.454-4.293$ & 0.560 \\
4.463 & $1.365-14.600$ & 0.013 \\
0.588 & $0.226-1.529$ & 0.276 \\
0.326 & $0.122-0.874$ & 0.026
\end{tabular}

image variation, that might result in overfitting for the training set $^{13,27,28}$. For this reason, we tested the performance of our algorithm in three, independent populations that vary in time of diagnosis, tissue preparation, and $\mathrm{H} \& \mathrm{E}$ staining investigated on 
Table 2 Clinicopathological data of the patients.

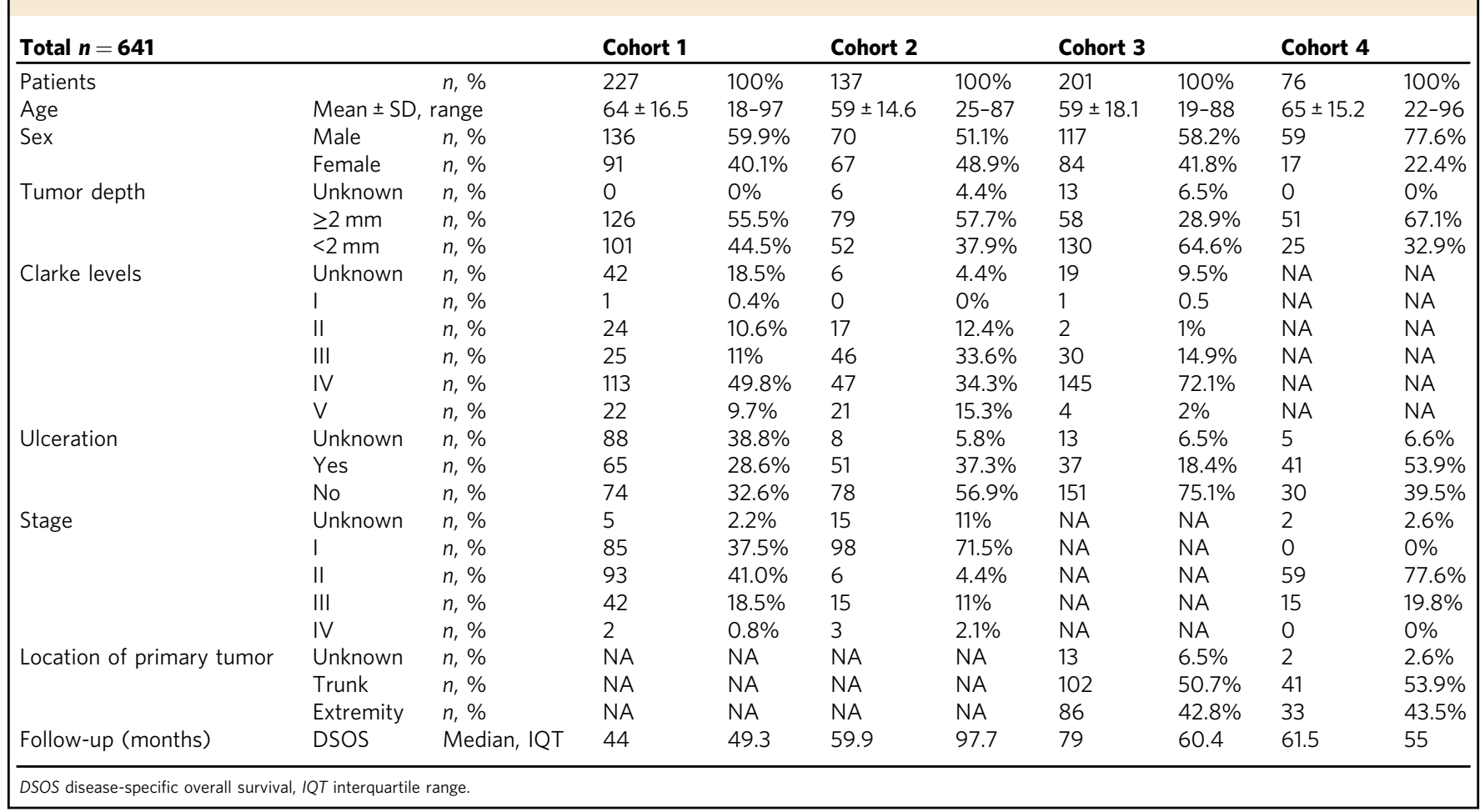

the oldest tumors dating back to the 1990s. While the treatments pre-dating immune therapy were approximately equivalent with respect to outcome, none of the cohorts are uniformly treated as would be seen in clinical trials. Another limitation is that all cases were scanned using a single scanner manufacturer, albeit at different institutions and at different magnifications. While the software algorithm NN192 appears to perform equivalently, it is not clear if the algorithm would perform similarly with images from other scanners. Further studies are needed to validate the prognostic potential of this algorithm in more independent cohorts and to determine the technical requirements for image acquisition. The performance of machine-learning-based classification depends on the training ${ }^{13}$. Even though this is an opensource software, and thus broadly available, quality control and systematic performance evaluations must be implemented prior to the use of eTIL\% scoring in adjuvant immunotherapy cohorts or in clinical practice. In our study, a pathologist performed quality control of the three algorithms to classify detected cells. Although we are not attempting to validate TMA technology here, it is notable that we found only modest correlation among TMA and whole-slide cases eTIL\% scores. This might be due to the fact that the analyzed tumor area on an average melanoma whole slide is $20-25$-fold larger than a TMA spot. However, eTIL $\%$ was prognostic irrespective of scoring on TMAs or wholeslide cases.

In conclusion, this study shows that an automated TIL score is a robust, independent prognostic marker in melanoma. With validation, we believe that this approach could be tested in the immunotherapy adjuvant setting to define a subset of patients that could potentially be spared treatment and its significant toxicities.

\section{Methods}

Patient cohorts and tissue preparation. Our retrospective collection of 641 melanoma tumors included four independent cohorts, three from Yale New Haven Hospital (YNHH), and one from Columbia University Irving Medical Center (CUMC). The training set (cohort \#1) consists of 227 patients diagnosed between
1993 and 2005 with 44 months median follow-up. The validation sets include cohort \#2: 137 patients diagnosed between 1999 and 2011 with 59.9 months median follow-up; cohort \#3: 201 patients diagnosed between 1981 and 2010 with 79 months median follow-up; and cohort \#4: 76 patients from CUMC, diagnosed between 2000 and 2012 with 61.5 months median follow-up (Table 2). Cohorts \#1, $\# 2$, and \#3 were assessed as tissue microarrays (TMAs). Representative tumor areas were selected by pathologists based on H\&E-stained slides. Duplicate cores (each $0.6 \mathrm{~mm}$ in diameter) were punched from each case. The H\&E-stained sections of the TMAs were scanned for analysis in this study. For cohorts \#2 and \#4, every field from the original H\&E slides were assessed resulting in average areas of assessment of $7.46 \mathrm{~mm}^{2}$ and $5.88 \mathrm{~mm}^{2}$ compared with $0.28 \mathrm{~mm}^{2}$ for TMA spots (Supplementary Fig. 3). One whole slide per patient selected by a pathologist was selected for the study. This study have complied with all relevant ethical regulations, and it was approved by the Yale Human Investigation Committee under protocol \#9505008219 and Columbia IRB protocol \#AAO2758. Patients in each cohort provided informed consent or (especially for older tissues) the tissue was obtained through Yale Human Investigation Committee protocol \#9505008219 which allows waiver of consent in some cases. Specifically, waiver of consent was in place for all patients at the time the Yale cohort \#1 collected in 2005. Older tissues in Yale Cohorts \#1 and \#3 were also collected with waiver of consent and for all deceased patients in all Yale cohorts. For the criterion standard, a pathologist scored TILs in cohort \#1 as absent, non-brisk, or brisk. The reporting recommendations for tumor marker prognostic studies (REMARK) were followed in our study ${ }^{29}$.

Digital-image analysis (DIA). In cohorts \#1, \#2, and \#3, the Aperio ScanScope XT platform (Leica Biosystems, Wetzlar, Germany) was used at $\times 20$ to digitize the slides with a pixel size of $0.4986 \mu \mathrm{m} \times 0.4986 \mu \mathrm{m}$. In cohort \#4, Aperio ScanScope XT platform (Leica Biosystems, Wetzlar, Germany) was used at $\times 40$ to digitize the slides with a pixel size of $0.2500 \mu \mathrm{m} \times 0.2500 \mu \mathrm{m}$. The QuPath open-source software platform ${ }^{30}$ was used to build automated TIL scoring algorithm. As the date of H\&E staining varied both between and within cohorts, we refined the H\&E stain estimates for each digitized slide (using the "estimate stain vectors" command in QuPath). We used watershed cell detection ${ }^{31}$ to segment the cells in the image with the following settings: Detection image: hematoxylin OD; requested pixel size: $0.5 \mu \mathrm{m}$; background radius: $8 \mu \mathrm{m}$; median filter radius: $0 \mu \mathrm{m}$; sigma: $1.5 \mu \mathrm{m}$; minimum cell area: $10 \mu \mathrm{m}^{2}$; maximum cell area: $400 \mu \mathrm{m}^{2}$; threshold: 0.1 ; maximum background intensity: 2 . The quality control of the cell segmentation was performed by a pathologist. In order to classify detected cells into tumor cells, immune cells (TILs), stromal cells, and others (false detections, background) (Fig. 3), we used neural network ${ }^{32}$ as a machine-learning method with eight hidden layers (maximum iterations: 100). The features used in the classification are described in Supplementary Table 2. In order to help the algorithm perform an accurate classification, we also added smoothed object features at $25 \mu \mathrm{m}$ and $50 \mu \mathrm{m}$ radius to supplement the existing measurements of individual cells. A number of rounds of 

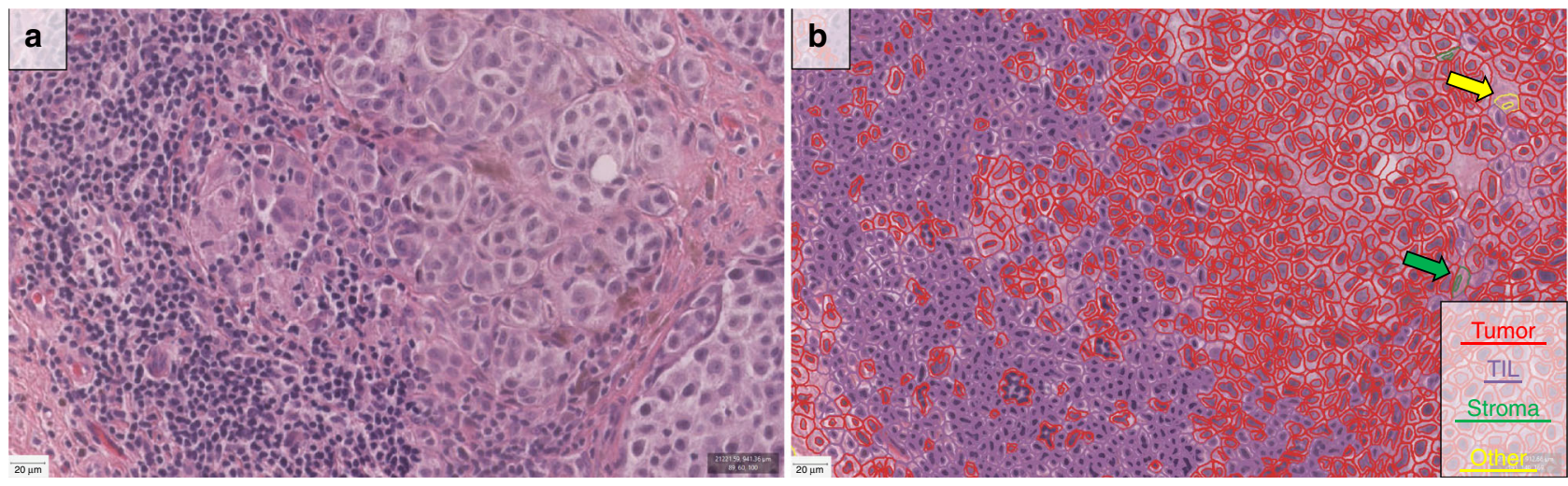

Fig. 3 Representative picture of a sample melanoma case showing the H\&E image (Zoom: $\times 20, \mathbf{a}$ ) and the digital-image analysis (DIA) mask (b). Scale bar represents $20 \mu \mathrm{m}$. Using the NN192 algorithm, segmentation shows red indicates tumor cells, purple marks immune cells, green corresponds to stromal cells, and yellow indicate others (false cell detections or unknown or background). Since stromal and "other" cells are rare, large arrows are included to show example cells.

optimization were required to achieve best results on the training set culminating in an algorithm called "NN192" that calculates the percentage of machine defined TILs calculated as follows: (TILs/TILs + Tumor cells) $\times 100$ called "eTIL\%". The quality control of the algorithm to classify detected cells was performed by a pathologist. In the whole-slide cohorts (cohorts \#2 and \#4), the analysis was run on the entire tumor as defined based on the pathologists' markings (Supplementary Fig. 3).

Statistical analysis. For statistical analysis, SPSS 22 software (IBM, Armonk, USA) software was used. The statistically significant cutoff for TILs scores was determined with X-tile software that uses outcome information to define thresh$\mathrm{old}^{33} \cdot X^{2}$ value was calculated for every possible division of the population and best cutoff (highest $\chi^{2}$ value) went under cross-validation to assess statistical significance by using the cut point derived from a training set to parse a separate validation set. Kaplan-Meier analysis supported with Log-rank test was executed to assess prognostic potential. To test independent prognostic potential, multivariate Cox-regression analysis was applied. Disease-specific overall survival (DSOS) was defined as the elapsed time from the date of primary diagnosis of the tumor to the date of death caused by melanoma, or when patients were last censored if died of non-melanoma cause or still alive. Mann-Whitney test was used to investigate the association between TILs scores and clinicopathological factors. To test the reproducibility between the corresponding TMA and whole-slide cases regarding TILs, intraclass correlation was supported by scatterplot and Wilcoxon signed-rank test. In all statistical analysis, the level of significance was set at $p<0.05$.

Reporting summary. Further information on research design is available in the Nature Research Reporting Summary linked to this article.

\section{Data availability}

The data is within the Article and Supplementary Information files and available from the authors upon request but the data from Columbia may require data transfer agreements. No personalized health information will be shared.

\section{Code availability}

Our TIL scoring algorithm for HE images of Melanoma has been deposited on GitHub: https://github.com/acsbal/Automated-TIL-scoring-QuPath-Classifier-for-Melanoma. The algorithm can be used in QuPath platform. The QuPath software may be downloaded at https://qupath.github.io/.

Received: 11 April 2019; Accepted: 15 October 2019; Published online: 29 November 2019

\section{References}

1. Disis, M. L. Immune regulation of cancer. J. Clin. Oncol. 28, 4531-4538 (2010).

2. Gajewski, T. F., Schreiber, H. \& Fu, Y. X. Innate and adaptive immune cells in the tumor microenvironment. Nat. Immunol. 14, 1014-1022 (2013).

3. Coulie, P. G., Van den, Eynde, van der Bruggen, B. J., P. \& Boon, T. Tumour antigens recognized by $\mathrm{T}$ lymphocytes: at the core of cancer immunotherapy. Nat. Rev. Cancer 14, 135-146 (2014).
4. Fridman, W. H., Pages, F., Sautes-Fridman, C. \& Galon, J. The immune contexture in human tumours: impact on clinical outcome. Nat. Rev. Cancer 12, 298-306 (2012).

5. Eggermont, A. M. et al. Prolonged survival in stage III melanoma with ipilimumab adjuvant therapy. N. Engl. J. Med. 375, 1845-1855 (2016).

6. Eggermont, A. M. M. et al. Adjuvant pembrolizumab versus placebo in resected stage III melanoma. N. Engl. J. Med. 378, 1789-1801 (2018).

7. Clark, W. H. Jr. et al. Model predicting survival in stage I melanoma based on tumor progression. J. Natl Cancer Inst. 81, 1893-1904 (1989).

8. Azimi, F. et al. Tumor-infiltrating lymphocyte grade is an independent predictor of sentinel lymph node status and survival in patients with cutaneous melanoma. J. Clin. Oncol. 30, 2678-2683 (2012).

9. Dieci, M. V. et al. Update on tumor-infiltrating lymphocytes (TILs) in breast cancer, including recommendations to assess TILs in residual disease after neoadjuvant therapy and in carcinoma in situ: a report of the International Immuno-Oncology Biomarker Working Group on Breast Cancer. Semin Cancer Biol. 52, 16-25 (2018).

10. Hendry, S. et al. Assessing tumor-infiltrating lymphocytes in solid tumors: a practical review for pathologists and proposal for a standardized method From the International Immunooncology Biomarkers Working Group: Part 1: assessing the host immune response, TILs in invasive breast carcinoma and ductal carcinoma in situ, metastatic tumor deposits and areas for further research. Adv. Anat. Pathol. 24, 235-251 (2017).

11. Hendry, S. et al. Assessing tumor-infiltrating lymphocytes in solid tumors: a practical review for pathologists and proposal for a standardized method from the International Immuno-oncology Biomarkers Working Group: part 2: TILs in melanoma, gastrointestinal tract carcinomas, non-small cell lung carcinoma and mesothelioma, endometrial and ovarian carcinomas, squamous cell carcinoma of the head and neck, genitourinary carcinomas, and primary brain tumors. Adv. Anat. Pathol. 24, 311-335 (2017).

12. Denkert, C. et al. Standardized evaluation of tumor-infiltrating lymphocytes in breast cancer: results of the ring studies of the international immuno-oncology biomarker working group. Mod. Pathol. 29, 1155-1164 (2016).

13. Klauschen, F. et al. Scoring of tumor-infiltrating lymphocytes: from visual estimation to machine learning. Semin Cancer Biol. 52, 151-157 (2018).

14. Wu, J., Zeng, P., Zhou, Y. \& Olivier, C. A novel color image segmentation method and its application to white blood cell image analysis. In 2006 8th International Conference on Signal Processing, (Fleet, D., Pajdla, T., Schiele, B. \& Tuytelaars, T. (eds)) Vol. 2 (IEEE, 2006) https://doi.org/10.1109/ ICOSP.2006.345700.

15. Zeiler M.D. \& Fergus R. Visualizing and Understanding Convolutional Networks. In: (Fleet, D., Pajdla, T., Schiele, B. \& Tuytelaars, T. (eds)) Computer Vision - ECCV 2014. ECCV 2014. Lecture Notes in Computer Science, vol. 8689 https://doi.org/10.1007/978-3-319-10590-1_53 (Springer, Cham 2014).

16. Wong, P. F. et al. Multiplex quantitative analysis of tumor-infiltrating lymphocytes and immunotherapy outcome in metastatic melanoma. Clin. Cancer Res. 25, 2442-2449 (2019).

17. Thomas, N. E. et al. Tumor-infiltrating lymphocyte grade in primary melanomas is independently associated with melanoma-specific survival in the population-based genes, environment and melanoma study. J. Clin. Oncol. 31, 4252-4259 (2013).

18. Larsen, T. E. \& Grude, T. H. A retrospective histological study of 669 cases of primary cutaneous malignant melanoma in clinical stage I. 3. The relation between the tumour-associated lymphocyte infiltration and age and sex, 
tumour cell type, pigmentation, cellular atypia, mitotic count, depth of invasion, ulceration, tumour type and prognosis. Acta Pathol. Microbiol. Scand. A 86a, 523-530 (1978).

19. Clemente, C. G. et al. Prognostic value of tumor infiltrating lymphocytes in the vertical growth phase of primary cutaneous melanoma. Cancer 77, 1303-1310 (1996).

20. Busam, K. J. et al. Histologic classification of tumor-infiltrating lymphocytes in primary cutaneous malignant melanoma. A study of interobserver agreement. Am. J. Clin. Pathol. 115, 856-860 (2001).

21. Masucci, G. V. et al. Validation of biomarkers to predict response to immunotherapy in cancer: Volume I - pre-analytical and analytical validation. J. Immunother. Cancer 4, 76 (2016).

22. Swiderska-Chadaj, Z. et al. Convolutional neural networks for lymphocyte detection in immunohistochemically stained whole-slide images. 1st Conference on Medical Imaging with Deep Learning, Amsterdam, The Netherlands (MIDL, 2018).

23. Garcia, E. et al. Automatic lymphocyte detection on gastric cancer ihc images using deep learning. In Computer-Based Medical Systems (CBMS), 2017 IEEE 30th International Symposium on 200-204 (IEEE, 2017).

24. Chen, T. \& Chefd'Hotel, C. Deep learning based automatic immune cell detection for immunohistochemistry images. In $\mathrm{Wu}$, Guorong, Zhang, Daoqiang, Zhou, Luping (eds)) International Workshop on Machine Learning in Medical Imaging 17-24 (Springer, 2014).

25. Galon, J. et al. Type, density, and location of immune cells within human colorectal tumors predict clinical outcome. Science 313, 1960-1964 (2006).

26. Heindl, A. et al. Relevance of spatial heterogeneity of immune infiltration for predicting risk of recurrence after endocrine therapy of ER+ breast cancer. J. Natl Cancer Inst. 110, 166-175 (2018).

27. Wienert, S. et al. Detection and segmentation of cell nuclei in virtual microscopy images: a minimum-model approach. Sci. Rep. 2, 503 (2012).

28. Wienert, S. et al. CognitionMaster: an object-based image analysis framework. Diagn. Pathol. 8, 34 (2013).

29. McShane, L. M. et al. Reporting recommendations for tumor marker prognostic studies (REMARK). J. Natl Cancer Inst. 97, 1180-1184 (2005).

30. Bankhead, P. et al. QuPath: open source software for digital pathology image analysis. Sci. Rep. 7, 16878 (2017).

31. Malpica, N. et al. Applying watershed algorithms to the segmentation of clustered nuclei. Cytometry 28, 289-297 (1997).

32. Bishop, C. M. Neural Networks for Pattern Recognition (Oxford University Press, 1995)

33. Camp, R. L., Dolled-Filhart, M. \& Rimm, D. L. X-tile: a new bio-informatics tool for biomarker assessment and outcome-based cut-point optimization. Clin. Cancer Res. 10, $7252-7259$ (2004).

\section{Acknowledgements}

Dr. B.A. was supported by the Fulbright Program and the Rosztoczy Foundation Scholarship Program. Dr. P.F.W. was supported by the Gruber Science Fellowship from the Gruber Foundation. This work was supported by Navigate BioPharma and grants from the NIH. Robyn Gartrell is supported by Swim Across America and the National
Center for Advancing Translational Sciences, National Institutes of Health, through Grant Number KL2TR001874.

\section{Author contributions}

Conception and design: B.A. and D.L.R. Case selection and clinical data collection: P.F.W R.D.G., J.S.P., E.M.R., Y.M.S., BG.R. and D.L.R. Acquisition of digital images: B.A., F.A., and S.G. Setting up image analysis algorithm, training of the algorithms, TIL scoring: B.A. Statistical analysis: B.A. Drafting of the paper: B.A., D.L.R. Critical revision of the paper: all authors. Final approval of the paper: all authors. Study supervision: D.L.R.

\section{Competing interests}

D.L.R. declares that he has served a consultant, advisor and/or servee on a Scientific Advisory Board for Amgen, Astra Zeneca, Agendia, Biocept, BMS, Cell Signaling Technology, Cepheid, Daiichi Sankyo, GSK, InVicro/Konica Minolta, Merck, NanoString, Perkin Elmer, PAIGE.AI, and Ultivue. He holds equity in PixelGear (start-up company related to direct tissue imaging) and Astra Zeneca, Cepheid, Navigate/Novartis, NextCure, Lilly, Ultivue, Ventana and Perkin Elmer/Akoya fund research in his lab. The remaining authors declare no competing interests.

\section{Additional information}

Supplementary information is available for this paper at https://doi.org/10.1038/s41467019-13043-2.

Correspondence and requests for materials should be addressed to D.L.R.

Peer review information Nature Communications thanks the anonymous reviewers for their contribution to the peer review of this work. Peer reviewer reports are available.

Reprints and permission information is available at http://www.nature.com/reprints

Publisher's note Springer Nature remains neutral with regard to jurisdictional claims in published maps and institutional affiliations.

Open Access This article is licensed under a Creative Commons Attribution 4.0 International License, which permits use, sharing, adaptation, distribution and reproduction in any medium or format, as long as you give appropriate credit to the original author(s) and the source, provide a link to the Creative Commons license, and indicate if changes were made. The images or other third party material in this article are included in the article's Creative Commons license, unless indicated otherwise in a credit line to the material. If material is not included in the article's Creative Commons license and your intended use is not permitted by statutory regulation or exceeds the permitted use, you will need to obtain permission directly from the copyright holder. To view a copy of this license, visit http://creativecommons.org/ licenses/by/4.0/

(C) The Author(s) 2019 